\title{
High expression of PIMREG predicts poor survival outcomes and is correlated with immune infiltrates in lung adenocarcinoma
}

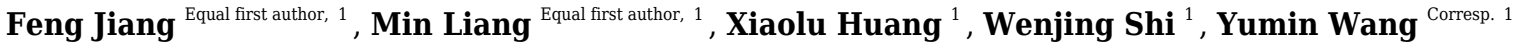 \\ ${ }^{1}$ Department of Laboratory Medicine, The First Affiliated Hospital of Wenzhou Medical University, Wenzhou,Zhe jiang, China \\ Corresponding Author: Yumin Wang \\ Email address: wangyumin0577@wmu.edu.cn
}

Background. PIMREG is upregulated in multiple cancer types. However, the potential role of PIMREG in lung adenocarcinoma (LUAD) remains unclear. The present study aimed to explore its clinical significance in LUAD. Methods. Using the Cancer Genome Atlas (TCGA) databases, we obtained 513 samples of LUAD and 59 normal samples from the Cancer Genome Atlas (TCGA) databases to analyze the relationship between PIMREG and LUAD. We used $t$ and Chi-square tests to evaluate the level of expression of PIMREG and its clinical implication in LUAD. The prognostic value of PIMREG in LUAD was identified through the Kaplan-Meier method, Cox regression analysis, and nomogram. Gene set enrichment analysis (GSEA) and single-sample gene set enrichment analysis (SSGSEA) were performed to screen biological pathways and analyze the correlation of the immune infiltrating level with the expression of PIMREG in LUAD. Results. PIMREG was highly expressed in patients with LUAD. Specifically, the level of PIMREG gradually increased from pathological stage I to IV. Further, we validated the higher expression of PIMREG expressed in LUAD cell lines. Moreover, PIMREG had a high diagnostic value, with an -AUC of 0.955 . Kaplan-Meier survival and Cox regression analyses revealed that the high expression of PIMREG was independently associated with poor clinical outcomes. In our prognostic nomogram, the expression of PIMREG implied a significant prognostic value. Gene set enrichment analysis (GSEA) identified that the high expression PIMREG phenotype was involved in the mitotic cell cycle, mRNA splicing, DNA repair, Rho GTPase signaling, TP53 transcriptional regulation, and translation pathways. Next, we also explored the correlation of PIMREG and tumor-immune interactions and found a negativecorrelationbetween PIMREG and the immune infiltrating level of T cells, macrophages, B cells, dendritic cells (DCs) , and CD8 ${ }^{+} \mathrm{T}$ cells in LUAD. Conclusions. High levels of PIMREG correlated with poor prognosis and immune infiltrates in LUAD. 
1 High expression of PIMREG predicts poor survival outcomes and is correlated with

2 immune infiltrates in lung adenocarcinoma

3 Feng Jiang ${ }^{1, *}$, Min Liang ${ }^{1,}$, Xiaolu Huang ${ }^{1}$, Wenjing Shi ${ }^{1}$, Yumin Wang ${ }^{1}$

$4{ }^{1}$ Department of Laboratory Medicine, The First Affiliated Hospital of Wenzhou Medical

5 University, Wenzhou 325000, China

$6 \quad$ *Feng Jiang and Min Liang contributed equally to this work.

7 Corresponding Author:

$8 \quad$ Yumin Wang

9 Department of Laboratory Medicine, First Affiliated Hospital of Wenzhou Medical University,

10 Nanbaixiang Street, Ouhai District, Wenzhou, Zhejiang Province 325000, China.

11 Email address: wangyumin@wmu.edu.cn

Background. PIMREG is upregulated in multiple cancer types. However, the potential role of PIMREG in lung adenocarcinoma (LUAD) remains unclear. The present study aimed to explore its clinical significance in LUAD.

Methods. Using the Cancer Genome Atlas (TCGA) databases, we obtained 513 samples of LUAD and 59 normal samples from the Cancer Genome Atlas (TCGA) databases to analyze the relationship between PIMREG and LUAD. We used $t$ and Chi-square tests to evaluate the level of expression of PIMREG and its clinical implication in LUAD. The prognostic value of PIMREG in LUAD was identified through the Kaplan-Meier method, Cox regression analysis, and nomogram. Gene set enrichment analysis (GSEA) and single-sample gene set enrichment analysis (ssGSEA) were performed to screen biological pathways and analyze the correlation of the immune infiltrating level with the expression of PIMREG in LUAD.

Results. PIMREG was highly expressed in patients with LUAD. Specifically, the level of PIMREG gradually increased from pathological stage I to IV. Further, we validated the higher expression of PIMREG expressed in LUAD cell lines. Moreover, PIMREG had a high diagnostic value, with an -AUC of 0.955. Kaplan-Meier survival and Cox regression analyses revealed that 
the high expression of PIMREG was independently associated with poor clinical outcomes. In our prognostic nomogram, the expression of PIMREG implied a significant prognostic value. Gene set enrichment analysis (GSEA) identified that the high expression PIMREG phenotype was involved in the mitotic cell cycle, mRNA splicing, DNA repair, Rho GTPase signaling, TP53 transcriptional regulation, and translation pathways. Next, we also explored the correlation of PIMREG and tumor-immune interactions and found a negative correlation between PIMREG and the immune infiltrating level of $\mathrm{T}$ cells, macrophages, $\mathrm{B}$ cells, dendritic cells (DCs), and CD8 ${ }^{+} \mathrm{T}$ cells in LUAD.

Conclusions. High levels of PIMREG correlated with poor prognosis and immune infiltrates in LUAD.

Subjects Bioinformatics, Oncology, Medical Genetics

Keywords PIMREG, Prognosis, Immune infiltrate, Lung adenocarcinoma

\section{INTRODUCTION}

Lung cancer is the most commonly occurring cancer in men and the third most commonly occurring cancer in women, resulting in the highest lethality rates among all cancers worldwide(Bray et al. 2018; Siegel et al. 2020). According to histopathological evaluations, 85\% of lung cancer cases are non-small cell lung cancer (NSCLC) (Latimer 2018). Of note, lung adenocarcinoma (LUAD) is the most common type of NSCLC (Chen et al. 2014; Meza et al. 2015). Despite significant breakthroughs in therapeutic interventions, such as surgery, chemotherapy, molecular targeted therapy, immunotherapy, and radiotherapy, the long-term outcomes of patients with LUAD at diagnosis remains unfavorable, and has been associated with a poor 5-y survival rate(AJW et al. 2019; Herbst et al. 2018). To improve the diagnosis and prognosis of LUAD, the identification of biomarkers and novel immune-related therapeutic targets is urgently needed.

PIMREG, PICALM interacting mitotic regulator (FAM64A), was first described as a clathrin assembly lymphoid myeloid leukemia gene (CALM) affects the subcellular localization of the leukemogenic fusion protein CALM/AF10(Archangelo et al. 2006). Furthermore, PIMREG has been reported to involve in the regulation of cell proliferation(Archangelo et al. 2008; Hashimoto et al. 2017; Hu et al. 2017; Zhang et al. 2014). In particular, PIMREG was shown to play a vital 
role in the enhancement of tumorigenesis and progression of neoplasms in multiple cancer types(Hu et al. 2017). Unfortunately, research on its function and the correlation of PIMREG with tumor immunity has not been reported in LUAD. Therefore, we first analyzed the transcription level and prognostic significance of PIMREG using data obtained from the Cancer Genome Atlas (TCGA) data. Moreover, we explored its biological mechanisms by GSEA analysis and further evaluated the association of PIMREG with the level of immune infiltration.

\section{MATERIALS AND METHODS}

\section{Patients and databases}

The gene expression data of 513 LUAD tissues and 59 normal tissues were downloaded from the TCGA database. Then, we specifically sorted the data of 57 pairs of LUAD and adjacent normal lung tissues. and further screened the clinicopathological characteristics and prognostic data of the cases obtained from TCGA. Unified processing of RNAseq data in TPM format of TCGA and GTEx. The expression of PIMREG were analyzed via GTEx database, and TCGA database. the expression of PIMREG of datasets of Hou et al., Landi et al and selamat et al. were analyzed via Oncomin database (https://www.oncomine.org). The HPA database was used to evaluate the protein expression of PIMREG in LUAD(Pontén et al. 2011).

\section{Gene Set Enrichment Analysis}

We used GSEA to identify gene sets and pathways associated with PIMREG based on transcriptional sequences from obtained TCGA. In this study, gene expression data were divided into high- and low- expressing PIMREG groups. We performed GSEA to compare these two groups and identify potential functions via the Broad Institute Website using the R package cluster profiler(Subramanian et al. 2005; Yu et al. 2012).

\section{Infiltration of immune cells}

To assess the relative abundance of the tumor tissue-infiltrating immune cells, we performed ssGSEA (single-sample gene set enrichment analysis). We used the "GSVA" (R package) and immune data-sets, including 24 types immunocytes analyze the infiltration level of immune cells in LUAD expression profile data(Bindea et al. 2013). Spearman correlation and the Wilcoxon rank sum test were used to analyze the correlation between PIMREG and these different immune cells, as well as the association of immune cells with the high- and low-expressing PIMRE groups G.

\section{Cell culture and quantitative real-time PCR}


The A549 and H1299 lung adenocarcinoma cell lines were purchased from the Cell Bank of the Chinese Academy of Sciences. Cells were cultured in 1640 medium supplemented with $10 \%$ fetal bovine serum (GIBCO, USA) at $37^{\circ} \mathrm{C}$ in a humidified $\mathrm{CO}_{2}$ incubator. The levels of expression levels of PIMREG were detected using an ABI 7500 Real-time PCR System. Relative expression levels were normalized to that of $\beta$-actin, which was used as an internal control. The primers used were as follows: PIMREG forward primer, 5'-CCTTAGTGGTGTCGGGGTCT-3'; reverse, 5'GAGGTCCCCATGTTCTGCCA-3'. $\beta$-actin forward primer: 5'-CCTGGCACCCAGCACAAT3'; reverse primer, 5'-GCTGATCCACATCTGCTGGAA-3'.

\section{Statistical analysis}

Statistical analysis of the expression of PIMREG in normal and LUAD groups was calculated using the Wilcoxon rank-sum test. We classified patients into two categories according to the "Median" expression of PIMREG. The clinicopathological features of PIMREG were analyzed using the Wilcoxon rank sum test or Kruskal-Wallis test and logistic regression. Prognostic analyses were performed using Kaplan-Meier survival analysis and Cox univariate and multivariate analyses. To evaluate the diagnostic significance of differentially expressed gene, a receiver operating characteristic (ROC) curve was generated using the "plotROC" package. The "rms" R package was adopted to plot the nomogram for the prognostic value among patients with LUAD. All statistical analyses were conducted in the R environment (v3.5.3) (http://www.rproject.org/). All figures were plotted using the R package ggplot2 (v3.1.0).

\section{RESULTS}

\section{Correlation analysis of expression of PIMREG in lung adenocarcinoma}

Table 1 lists the expression of PIMREG and clinical data of 513 patients with LUAD downloaded from TCGA. We observed a significant relationship between the expression of PIMREG and clinicopathological parameters, such as T stage, N stage, pathologic stage, sex, smoking, tumor status, number of packs smoked per year, and age. In addition, we found that patients with high expression of PIMREG presented with elevated mutations in TP53 $(\mathrm{p}<0.001)$ compared with those having a low expression of PIMREG (See Table 1).

\section{Aberrant upregulation of PIMREG in lung adenocarcinoma}

We used the TCGA database to reveal the mRNA expression pattern of PIMREG in LUAD tissues and normal tissues. We also assessed the expression levels of PIMREG in LUAD and normal 
121 tissues using Oncomine, GTEx, and HPA database. PIMREG expression was increased at the 122 mRNA and protein levels (Fig. 1S). The average expression level of PIMREG mRNA was dramatically higher in LUAD tissues than in normal samples $(p<0.001$ Fig. $1 \mathrm{~A})$. We then analyzed the expression of PIMREG in paired tumor and adjacent samples (Fig. 1B). We accordingly identified a significant difference, with tumor tissues exhibiting an increase in the expression of PIMREG. To evaluate the level of expression of PIMREG in various cancers, we performed a systematic analysis using data downloaded from TCGA databases. Our results showed that PIMREG was overexpressed in many tumor types, including bladder urothelial carcinoma, bone cancer, cholangiocarcinoma, and glioblastoma multiforme (Fig. 1C). Meanwhile, we observed that as the mRNA expression of PIMREG increased, the risk of pathological stage was also increased (Fig. 2A). In addition, we found that T stage $(\mathrm{p}<0.001)$, N stage $(\mathrm{p}<0.001), \mathrm{M}$ stage $(\mathrm{p}=0.065)$, TP53 status ( $\mathrm{p}<0.001)$, and smoking history $(\mathrm{p}<0.001)$ were also significantly associated with the mRNA expression of PIMREG. To confirm the effect of the function of PIMREG in LUAD, we accessed the effect of the expression of PIMREG on the survival rate of patients with LUAD. Our results revealed that upregulation of PIMREG was linked to worse overall survival (OS), disease-specific survival (DSS), and progression-free interval (PFI) in patients with LUAD (Fig. 3). Moreover, we performed receiver operating characteristic (ROC) analysis of LUAD to reveal the diagnostic value of $\mathrm{ABC}$, and found an area under the curve (AUC) value of 0.955 .

\section{Univariate and multivariate analyses of survival}

To further explore the risk factors in patients with LUAD, we performed univariate and multivariate analyses. We noticed that the univariate analysis using the COX regression model showed that $\mathrm{T}$ stage, $\mathrm{M}$ stage, $\mathrm{N}$ stage, pathologic stage, primary therapy outcome, residual tumor and tumor status were associated with OS $(\mathrm{p}=0.03, \mathrm{p}<0.001, \mathrm{p}=0.007, \mathrm{p}<0.001, \mathrm{p}<0.001, \mathrm{p}$ $<0.001, \mathrm{p}<0.001$, and $\mathrm{p}<0.001$, respectively) (Table 2). In addition, we detected that the expression of PIMREG (hazard ratio $(\mathrm{HR})=1.755$, $95 \%$ confidence interval (CI) 1.304-2.361, Cox $\mathrm{p}<0.001$ ) was a significant predictor of OS. We then performed multivariate analysis, which revealed the independent risk factors. We found that the primary therapy outcome and tumor status were independent prognostic factors of OS inpatients with LUAD. However, we observed that the expression of PIMREG was still independently associated with OS (HR $=1.870,95 \%$ CI 1.085 3.223, Cox $\mathrm{p}=0.024)$. Furthermore, we validated the high expression of PIMREG in PFI and DSS (Supplementary Table 1,2). Therefore, the high expression of PIMREG was demonstrated to be a 
152 good predictor of poor OS, DSS, and PFI in patients with LUAD.

153 Subgroup analysis

154 We further performed a subgroup analysis to assess the impact of the expression of PIMREG on

155 OS according to age, sex, and anatomic neoplasm subdivision risk factors. We found that in each

156

157

158

159

160

161

162

163

164

165

166

167

168

169

170

171

172

173

174

175

176

177

178

179

180

181

182 and every subgroup stratified by age, sex, and anatomic neoplasm subdivision, the high expression of PIMREG continued to lead to poor survival (Fig. 4).

\section{Construction of nomogram}

The above results suggested that the level of expression of PIMREG, primary therapy outcome and tumor status in patients with LUAD might be linked to prognosis. Therefore, we constructed a prognostic nomogram to predict individual survival probability through the levels of expression of PIMREG, primary therapy outcome and tumor status (Fig. 5A). The calibration curve of the prediction model showed that the established lines of 1-, 3- and 5-y survival matched the ideal line at a high degree (Fig. 5B). We observed that the $\mathrm{C}$ index of our prognostic nomogram reached $0.758(0.734-0.782)$, indicating that the model had a reliable potential for the prediction of overall survival.

\section{Gene set enrichment analysis identified identities an PIMREG-related signaling pathway}

We next conducted GSEA to identify differences in the signaling pathways between low- and highexpression PIMREG data-set (Fig. 6). We respectively found that PIMREG was related to the mitotic cell cycle, mRNA splicing, DNA repair, Rho GTPase signaling, TP53 transcriptional regulation, translation pathways. Furthermore, GSEA revealed that the high level of PIMREG might be linked to cancer-promoting pathways.

\section{Immune infiltration analysis}

To understand the underlying cause of worse prognosis in patients with LUAD with immune infiltration, we carried out an enrichment analysis of the LUAD tumor microenvironment using ssGSEA. We found that the expression of PIMREG was negatively correlated with T cells, B cells, dendritic cells (DCs), macrophages, and $\mathrm{CD}^{+} \mathrm{T}$ cells (Fig. 7).

\section{Correlation analysis of expression of PIMREG in lung adenocarcinoma}

In order to evaluate the potential utility of PIMREG as a biomarker of LUAD, we further verified the expression of PIMREG in cell lines using qPCR (Fig. 8). We found that the mRNA level of PIMREG in the A549 and H1299 LUAD cell lines was increased 4.26- and 2.87-fold compared with that in 2B the normal lung epithelial cell line, which was consistent with our above-mentioned 
results.

\section{Discussion}

In this study, we found that the PIMREG gene was overexpressed in tumor compared with normal and adjacent normal tissues, and this overexpression was linked to worse OS, DSS and PFI. ROC analysis confirmed that PIMREG could be used as a biomarker for the prognosis of LUAD, while univariate and multivariate Cox analyses provided evidence that the mRNA expression of PIMREG might be an independent prognostic indicator of LUAD. Moreover, the level of expression of PIMREG was positivity correlated with the pathological stage. Patients with a high level of PIMREG were more likely to be presented with the disease in a late pathological stage, suggesting PIMREG as a tumor-related gene in LUAD. Furthermore, our prognostic nomogram exhibited satisfactory potential for clinical application.

Of note, the expression of PIMREG has been confirmed in several tumors(Archangelo et al. 2006; Barbutti et al. 2016). Using data obtained from TCGA databases, Jiao et al showed that PIMREG was overexpressed in pancreatic cancer and related to poor outcomes; however no further experiments have been performed to verify this finding(Jiao et al. 2019). In addition, Yamada et al evaluated the prognostic value of the survival-associated gene PIMREG in breast cancer and renal cell carcinoma(Yamada et al. 2018). Yao et al also found that the expression of PIMREG was significantly associated with breast cancer, and patients with high expression of PIMREG were associated with poor prognosis. Further analysis validated this observation by demonstrating that PIMREG promoted the migration and proliferation of breast cancer cells(Yao et al. 2019). However, its level of expression and prognosis in LUAD have not been previously reported. Consistently, the current study demonstrated the high expression of PIMREG and its correlation with poor survival in patients with LUAD. In addition, we further found that the high expression of PIMREG continued to lead to poor survival even when patients where stratified according to age, sex, and anatomic neoplasm subdivision.

Prior studies have reported that higher expression of PIMREG promotes cell proliferation(Archangelo et al. 2008; Yao et al. 2019). Whereas, silencing of PIMREG was reported to repress cell cycle-promoting genes in fetal cardiomyocytes(Hashimoto et al. 2017). Our results showed that the underlying molecular mechanisms through which $\mathrm{ABC}$ exerts its effect might be related to the cell cycle, mRNA splicing, DNA repair, Rho GTPase signaling, TP53 
214 transcriptional regulation, and translation, as identified by GSEA.

215 In recent years, the role of immune cell infiltration in the development and progression of 216 cancer has attracted increasing attention(Camidge et al. 2019; Carbone et al. 2015). Most studies

217 have shown that infiltration of T and B cells in NSCLC predicts a favorable outcome(Edlund et al. 218 2019; Wang et al. 2019b; Zhang et al. 2019). High expression of $\mathrm{CD}^{+} \mathrm{T}$ cells has been 219 demonstrated to predict increased survival in LUAD(Iglesia et al. 2016; Wang et al. 2020). In 220 addition, macrophages have been reported to play an important role in regulating tumor innate and 221 acquired immunity. For instance, the M1, CD204M2, and CD68 macrophages have been 222 considered to confer protective immunity against several factors in the tumor microenvironment 223 of NSCLC(Rakaee et al. 2019). However, macrophages are also known to promote 224 tumor progression, Therefore, the role of the infiltration and activation of macrophages in cancer 225 is not clear (Bercovici et al. 2019). Previous studies have also found that dendritic cells (DC) 226 presented antigens to activate anti-tumor T cells(Wculek et al. 2020). Some studies demonstrated that the infiltration of DCs was related to protective immunity in LUAD(Wang et al. 2019a). In our study, we found that the expression of PIMREG was negatively correlated with $\mathrm{T}$ cells, macrophages, B cells, DCs and $\mathrm{CD}^{+} \mathrm{T}$ cells in LUAD, indicating that PIMREG might plays a significant role in regulating the tumor immune microenvironment. However, the mechanism by which PIMREG affects the tumor immune microenvironment and tumor progression in LUAD remains unclear. Further basic and clinical experiments are necessary to comprehensively elucidate the biological impact of PIMREG in lung cancer.

\section{CONCLUSIONS}

Overall, the high expression of PIMREG was correlated with prognostic implications. Moreover, PIMREG was a negatively correlated with T cells, B cells, macrophages, DCs and CD8 ${ }^{+}$ T cells. Therefore, PIMREG, which might be associated with immune infiltration, might serve as a prognostic factor in patients with LUAD.

\section{REFERENCES}

AJW G, H L, A DS, RA T, AA E, S O, DG B, and WY C. 2019. Comparison of Clinical Characteristics and Outcomes in Relapsed Versus De Novo Metastatic Non-Small Cell Lung Cancer. American journal of clinical oncology 42:75-81. 10.1097/coc.0000000000000483

Archangelo LF, Gläsner J, Krause A, and Bohlander SK. 2006. The novel CALM interactor CATS influences the subcellular localization of the leukemogenic fusion protein CALM/AF10. Oncogene 25:4099-4109. 10.1038/sj.onc. 1209438

Archangelo LF, Greif PA, Hölzel M, Harasim T, Kremmer E, Przemeck GK, Eick D, Deshpande AJ, Buske C, de 
Angelis MH, Saad ST, and Bohlander SK. 2008. The CALM and CALM/AF10 interactor CATS is a marker for proliferation. Mol Oncol 2:356-367. 10.1016/j.molonc.2008.08.001

Barbutti I, Xavier-Ferrucio JM, Machado-Neto JA, Ricon L, Traina F, Bohlander SK, Saad ST, and Archangelo LF. 2016. CATS (FAM64A) abnormal expression reduces clonogenicity of hematopoietic cells. Oncotarget 7:68385-68396. 10.18632/oncotarget.11724

Bercovici N, Guérin MV, Trautmann A, and Donnadieu E. 2019. The Remarkable Plasticity of Macrophages: A Chance to Fight Cancer. Front Immunol 10:1563. 10.3389/fimmu.2019.01563

Bindea G, Mlecnik B, Tosolini M, Kirilovsky A, Waldner M, Obenauf AC, Angell H, Fredriksen T, Lafontaine L, Berger A, Bruneval P, Fridman WH, Becker C, Pagès F, Speicher MR, Trajanoski Z, and Galon J. 2013. Spatiotemporal dynamics of intratumoral immune cells reveal the immune landscape in human cancer. Immunity 39:782-795. 10.1016/j.immuni.2013.10.003

Bray F, Ferlay J, Soerjomataram I, Siegel RL, Torre LA, and Jemal A. 2018. Global cancer statistics 2018: GLOBOCAN estimates of incidence and mortality worldwide for 36 cancers in 185 countries. Ca Cancer $J$ Clin 68:394-424. 10.3322/caac.21492

Camidge DR, Doebele RC, and Kerr KM. 2019. Comparing and contrasting predictive biomarkers for immunotherapy and targeted therapy of NSCLC. Nat Rev Clin Oncol 16:341-355. 10.1038/s41571-019-0173-9

Carbone DP, Gandara DR, Antonia SJ, Zielinski C, and Paz-Ares L. 2015. Non-Small-Cell Lung Cancer: Role of the Immune System and Potential for Immunotherapy. $J$ Thorac Oncol 10:974-984. $10.1097 /$ jto.0000000000000551

Chen Z, Fillmore CM, Hammerman PS, Kim CF, and Wong KK. 2014. Non-small-cell lung cancers: a heterogeneous set of diseases. Nat Rev Cancer 14:535-546. 10.1038/nrc3775

Edlund K, Madjar K, Mattsson JSM, Djureinovic D, Lindskog C, Brunnström H, Koyi H, Brandén E, Jirström K, Pontén F, Rahnenführer J, Micke P, and Hengstler JG. 2019. Prognostic Impact of Tumor Cell Programmed Death Ligand 1 Expression and Immune Cell Infiltration in NSCLC. $J$ Thorac Oncol 14:628-640. 10.1016/j.jtho.2018.12.022

Hashimoto K, Kodama A, Honda T, Hanashima A, Ujihara Y, Murayama T, Nishimatsu SI, and Mohri S. 2017. Fam64a is a novel cell cycle promoter of hypoxic fetal cardiomyocytes in mice. Sci Rep 7:4486. 10.1038/s41598-017-04823-1

Herbst RS, Morgensztern D, and Boshoff C. 2018. The biology and management of non-small cell lung cancer. Nature 553:446-454. 10.1038/nature25183

Hu S, Yuan H, Li Z, Zhang J, Wu J, Chen Y, Shi Q, Ren W, Shao N, and Ying X. 2017. Transcriptional response profiles of paired tumor-normal samples offer novel perspectives in pan-cancer analysis. Oncotarget 8:41334-41347. 10.18632/oncotarget.17295

Iglesia MD, Parker JS, Hoadley KA, Serody JS, Perou CM, and Vincent BG. 2016. Genomic Analysis of Immune Cell Infiltrates Across 11 Tumor Types. J Natl Cancer Inst 108. 10.1093/jnci/djw144

Jiao Y, Fu Z, Li Y, Zhang W, and Liu Y. 2019. Aberrant FAM64A mRNA expression is an independent predictor of poor survival in pancreatic cancer. PLoS One 14:e0211291. 10.1371/journal.pone.0211291

Latimer KM. 2018. Lung Cancer: Clinical Presentation and Diagnosis. FP Essent 464:23-26.

Meza R, Meernik C, Jeon J, and Cote ML. 2015. Lung cancer incidence trends by gender, race and histology in the United States, 1973-2010. PLoS One 10:e0121323. 10.1371/journal.pone.0121323

Pontén F, Schwenk JM, Asplund A, and Edqvist PH. 2011. The Human Protein Atlas as a proteomic resource for 
biomarker discovery. J Intern Med 270:428-446. 10.1111/j.1365-2796.2011.02427.x

Rakaee M, Busund LR, Jamaly S, Paulsen EE, Richardsen E, Andersen S, Al-Saad S, Bremnes RM, Donnem T, and Kilvaer TK. 2019. Prognostic Value of Macrophage Phenotypes in Resectable Non-Small Cell Lung Cancer Assessed by Multiplex Immunohistochemistry. Neoplasia 21:282-293. 10.1016/j.neo.2019.01.005

Siegel RL, Miller KD, and Jemal A. 2020. Cancer statistics, 2020. Ca Cancer J Clin 70:7-30. 10.3322/caac.21590

Subramanian A, Tamayo P, Mootha VK, Mukherjee S, Ebert BL, Gillette MA, Paulovich A, Pomeroy SL, Golub TR, Lander ES, and Mesirov JP. 2005. Gene set enrichment analysis: a knowledge-based approach for interpreting genome-wide expression profiles. Proc Natl Acad Sci U S A 102:15545-15550. 10.1073/pnas.0506580102

Wang JB, Huang X, and Li FR. 2019a. Impaired dendritic cell functions in lung cancer: a review of recent advances and future perspectives. Cancer Commun (Lond) 39:43. 10.1186/s40880-019-0387-3

Wang SS, Liu W, Ly D, Xu H, Qu L, and Zhang L. 2019b. Tumor-infiltrating B cells: their role and application in anti-tumor immunity in lung cancer. Cell Mol Immunol 16:6-18. 10.1038/s41423-018-0027-x

Wang W, Ren S, Wang Z, Zhang C, and Huang J. 2020. Increased expression of TTC21A in lung adenocarcinoma infers favorable prognosis and high immune infiltrating level. Int Immunopharmacol 78:106077. 10.1016/j.intimp.2019.106077

Wculek SK, Cueto FJ, Mujal AM, Melero I, Krummel MF, and Sancho D. 2020. Dendritic cells in cancer immunology and immunotherapy. Nat Rev Immunol 20:7-24. 10.1038/s41577-019-0210-z

Yamada Y, Arai T, Kojima S, Sugawara S, Kato M, Okato A, Yamazaki K, Naya Y, Ichikawa T, and Seki N. 2018. Regulation of antitumor miR-144-5p targets oncogenes: Direct regulation of syndecan-3 and its clinical significance. Cancer Sci 109:2919-2936. 10.1111/cas.13722

Yao Z, Zheng X, Lu S, He Z, Miao Y, Huang H, Chu X, Cai C, and Zou F. 2019. Knockdown of FAM64A suppresses proliferation and migration of breast cancer cells. Breast Cancer 26:835-845. 10.1007/s12282-019-00991-2

Yu G, Wang LG, Han Y, and He QY. 2012. clusterProfiler: an R package for comparing biological themes among gene clusters. Omics 16:284-287. 10.1089/omi.2011.0118

Zhang C, Han Y, Huang H, Min L, Qu L, and Shou C. 2014. Integrated analysis of expression profiling data identifies three genes in correlation with poor prognosis of triple-negative breast cancer. Int J Oncol 44:2025-2033. 10.3892/ijo.2014.2352

Zhang J, Endres S, and Kobold S. 2019. Enhancing tumor T cell infiltration to enable cancer immunotherapy. Immunotherapy 11:201-213. 10.2217/imt-2018-0111 


\section{Table $\mathbf{1}$ (on next page)}

Correlation between PIMREG and clinicopathological characteristics 
Table 1 Correlation between PIMREG and clinicopathological characteristics.

\begin{tabular}{|c|c|c|c|c|}
\hline \multirow{3}{*}{ Characters } & \multirow{3}{*}{ level } & \multicolumn{2}{|r|}{ High } & \multirow{3}{*}{$\mathrm{p}$} \\
\hline & & expression of & expression of & \\
\hline & & PIMREG & PIMREG & \\
\hline $\mathrm{n}$ & & 257 & 256 & \\
\hline \multirow[t]{4}{*}{ T stage $(\%)$} & $\mathrm{T} 1$ & $102(40.0 \%)$ & $66(25.9 \%)$ & 0.003 \\
\hline & $\mathrm{T} 2$ & $118(46.3 \%)$ & $158(62.0 \%)$ & \\
\hline & $\mathrm{T} 3$ & $25(9.8 \%)$ & $22(8.6 \%)$ & \\
\hline & $\mathrm{T} 4$ & $10(3.9 \%)$ & $9(3.5 \%)$ & \\
\hline \multirow[t]{4}{*}{$\mathrm{N}$ stage $(\%)$} & No & $180(72.9 \%)$ & $150(59.1 \%)$ & 0.005 \\
\hline & N1 & $40(16.2 \%)$ & $55(21.7 \%)$ & \\
\hline & $\mathrm{N} 2$ & $26(10.5 \%)$ & $48(18.9 \%)$ & \\
\hline & N3 & $1(0.4 \%)$ & $1(0.4 \%)$ & \\
\hline \multirow[t]{2}{*}{ M stage $(\%)$} & M0 & $172(95.0 \%)$ & $172(91.5 \%)$ & 0.252 \\
\hline & M1 & $9(5.0 \%)$ & $16(8.5 \%)$ & \\
\hline \multirow[t]{4}{*}{ Pathologic stage (\%) } & Stage I & $156(61.9 \%)$ & $118(46.6 \%)$ & 0.006 \\
\hline & Stage II & $53(21.0 \%)$ & $68(26.9 \%)$ & \\
\hline & Stage III & $33(13.1 \%)$ & $51(20.2 \%)$ & \\
\hline & Stage IV & $10(4.0 \%)$ & $16(6.3 \%)$ & \\
\hline \multirow[t]{4}{*}{ Primary therapy outcome $(\%)$} & $\mathrm{CR}$ & $164(74.9 \%)$ & $151(72.9 \%)$ & 0.159 \\
\hline & PD & $28(12.8 \%)$ & $40(19.3 \%)$ & \\
\hline & PR & $4(1.8 \%)$ & $2(1.0 \%)$ & \\
\hline & $\mathrm{SD}$ & $23(10.5 \%)$ & $14(6.8 \%)$ & \\
\hline \multirow[t]{2}{*}{ Gender (\%) } & Female & $159(61.9 \%)$ & $117(45.7 \%)$ & $<0.001$ \\
\hline & Male & $98(38.1 \%)$ & $139(54.3 \%)$ & \\
\hline \multirow[t]{2}{*}{ Race (\%) } & Asian & $5(2.2 \%)$ & $2(0.9 \%)$ & 0.657 \\
\hline & Black or African & $26(11.4 \%)$ & $26(11.9 \%)$ & \\
\hline
\end{tabular}




\begin{tabular}{|c|c|c|c|c|}
\hline \multicolumn{4}{|c|}{ American } & \multirow{5}{*}{0.063} \\
\hline \multirow{3}{*}{ Residual tumor (\%) } & White & $197(86.4 \%)$ & $190(87.2 \%)$ & \\
\hline & R0 & $162(93.6 \%)$ & $182(96.8 \%)$ & \\
\hline & $\mathrm{R} 1$ & $10(5.8 \%)$ & $3(1.6 \%)$ & \\
\hline \multirow{3}{*}{$\begin{array}{l}\text { Anatomic neoplasm } \\
\text { subdivision (\%) }\end{array}$} & $\mathrm{R} 2$ & $1(0.6 \%)$ & $3(1.6 \%)$ & \\
\hline & Left & $97(39.0 \%)$ & $102(41.0 \%)$ & 0.714 \\
\hline & Right & $152(61.0 \%)$ & $147(59.0 \%)$ & \\
\hline \multirow[t]{2}{*}{ Anatomic neoplasm } & & & & \\
\hline & Central Lung & $29(34.1 \%)$ & $33(31.7 \%)$ & 0.848 \\
\hline subdivision $2(\%)$ & Peripheral Lung & $56(65.9 \%)$ & $71(68.3 \%)$ & \\
\hline \multirow[t]{2}{*}{ Smoker (\%) } & No & $50(20.2 \%)$ & $24(9.6 \%)$ & 0.001 \\
\hline & Yes & $198(79.8 \%)$ & $227(90.4 \%)$ & \\
\hline \multirow[t]{2}{*}{ Tumor status (\%) } & Tumor free & $158(69.0 \%)$ & $130(56.8 \%)$ & 0.009 \\
\hline & With tumor & $71(31.0 \%)$ & $99(43.2 \%)$ & \\
\hline \multirow[t]{2}{*}{ TP53 status (\%) } & Mut & $76(29.8 \%)$ & $165(65.2 \%)$ & $<0.001$ \\
\hline & WT & $179(70.2 \%)$ & $88(34.8 \%)$ & \\
\hline \multirow[t]{2}{*}{ KRAS status (\%) } & Mut & $66(25.9 \%)$ & $73(28.9 \%)$ & 0.515 \\
\hline & WT & $189(74.1 \%)$ & $180(71.1 \%)$ & \\
\hline \multicolumn{2}{|c|}{ Age (mean (SD)) } & $66.67(10.01)$ & $63.91(9.88)$ & 0.002 \\
\hline \multicolumn{2}{|c|}{ number pack years smoked (mean (SD)) } & $38.26(27.02)$ & $44.60(27.03)$ & 0.029 \\
\hline
\end{tabular}


Table 2 (on next page)

Univariate analysis and multivariate analysis of the correlation between clinicopathological characteristics and OS in LUAD 
1 Table 2 Univariate analysis and multivariate analysis of the correlation between clinicopathological characteristics 2 and OS in LUAD

\begin{tabular}{|c|c|c|c|c|c|}
\hline Characteristics & $\begin{array}{c}\text { Total }(\mathrm{N} \\
)\end{array}$ & $\begin{array}{c}\mathrm{HR}(95 \% \mathrm{CI}) \\
\text { Univariate } \\
\text { analysis }\end{array}$ & $\begin{array}{l}\mathrm{P} \text { value } \\
\text { Univariat } \\
\text { e analysis }\end{array}$ & $\begin{array}{c}\text { HR(95\% CI }) \\
\text { Multivariate } \\
\text { analysis }\end{array}$ & $\begin{array}{c}\text { P value } \\
\text { Multivariate } \\
\text { analysis }\end{array}$ \\
\hline T stage (T2\&T3\&T4 vs. T1) & 501 & $\begin{array}{c}1.668(1.184- \\
2.349)\end{array}$ & 0.003 & $1.406(0.737-2.685)$ & 0.301 \\
\hline N stage (N1\&N2\&N3 vs. N0) & 492 & $\begin{array}{c}2.606(1.939- \\
3.503)\end{array}$ & $<0.001$ & $1.597(0.691-3.693)$ & 0.274 \\
\hline M stage (M1 vs. M0) & 360 & $\begin{array}{l}2.111(1.232- \\
3.616)\end{array}$ & 0.007 & $1.023(0.377-2.775)$ & 0.964 \\
\hline $\begin{array}{l}\text { Pathologic stage (Stage II\&Stage } \\
\text { III\&Stage IV vs. Stage I) }\end{array}$ & 496 & $\begin{array}{c}2.975(2.188- \\
4.045)\end{array}$ & $<0.001$ & $0.748(0.302-1.852)$ & 0.53 \\
\hline $\begin{array}{l}\text { Primary therapy outcome } \\
\text { (PD\&SD\&PR vs. CR) }\end{array}$ & 419 & $\begin{array}{c}2.818(2.004- \\
3.963)\end{array}$ & $<0.001$ & $2.486(1.442-4.286)$ & 0.001 \\
\hline Residual tumor (R1\&R2 vs. R0) & 352 & $\begin{array}{c}3.973(2.217- \\
7.120)\end{array}$ & $<0.001$ & $1.957(0.723-5.294)$ & 0.186 \\
\hline Gender (Male vs. Female) & 504 & $\begin{array}{c}1.060(0.792- \\
1.418)\end{array}$ & 0.694 & & \\
\hline Age $(>65$ vs. $<=65)$ & 494 & $\begin{array}{c}1.228(0.915- \\
1.649)\end{array}$ & 0.171 & & \\
\hline $\begin{array}{c}\text { Race (White vs. Asian\&Black or } \\
\text { African American) }\end{array}$ & 446 & $\begin{array}{c}1.422(0.869- \\
2.327)\end{array}$ & 0.162 & & \\
\hline $\begin{array}{l}\text { Anatomic neoplasm subdivision } \\
\text { (Right vs. Left) }\end{array}$ & 490 & $\begin{array}{c}1.024(0.758- \\
1.383)\end{array}$ & 0.878 & & \\
\hline $\begin{array}{c}\text { Anatomic neoplasm } \\
\text { subdivision2 (Peripheral Lung }\end{array}$ & 182 & $\begin{array}{c}0.913(0.570- \\
1.463)\end{array}$ & 0.706 & & \\
\hline
\end{tabular}


vs. Central Lung)

number pack years smoked

$(>=40$ vs. $<40)$

Smoker (Yes vs. No)

Tumor status (With tumor vs.

Tumor free)

TP53 status (Mut vs. WT)

KRAS status (Mut vs. WT)

PIMREG (High vs. Low)

$\begin{array}{ccc} & 1.038(0.723- & \\ & 1.490) & \\ & & \\ 490 & 0.887(0.587- & \\ & 1.339) & \end{array}$

450

$6.211(4.258$

9.059)

$<0.001$

5.942(3.282-

$10.756)$

$<0.001$

1.254(0.936-

499

1.680)

1.087(0.779-

499

$$
1.517)
$$

1.755(1.304-

504
0.13

0.623

$<0.001$

$1.870(1.085-3.223)$
0.024

\subsection{1)}

3 


\section{Figure 1}

The level of PIMREG expression in the mRNA based on TCGA database.

(A) Comparison of PIMREG expression level between LUAD tissues and normal tissue. (B) PIMREG expression level in 57 matched LUAD tissues and corresponding normal tissues. (C) Overview of PIMREG mRNA expression in different tumor tissues and adjacent normal tissues. BLCA, Bladder urothelial carcinoma; BRCA, Breast invasive carcinoma; CESC, Cervical squamous cell carcinoma and endocervical adenocarcinoma; $\mathrm{CHOL}$, Cholangiocarcinoma; COAD, Colon adenocarcinoma; ESCA, Esophageal carcinoma; GBM, Glioblastomamultiform; HNSC, Head and neck squamous cell carcinoma; KIRC, Kidneyrenal clearcell carcinoma; KIRP, Kidneyrenalclearcell carcinoma; LIHC, Liver hepatocellular carcinoma; LUAD, lung adenocarcinoma ; LUSC, lung squamous cell carcinoma; PRAD, Prostate adenocarcinoma; READ, rectal adenocarcinoma; SARC, Sarcoma; STAD, Stomach adenocarcinoma; THCA, Thyroid carcinoma; UCEC, Uterine Corpus Endometrial Carcinoma. 
A

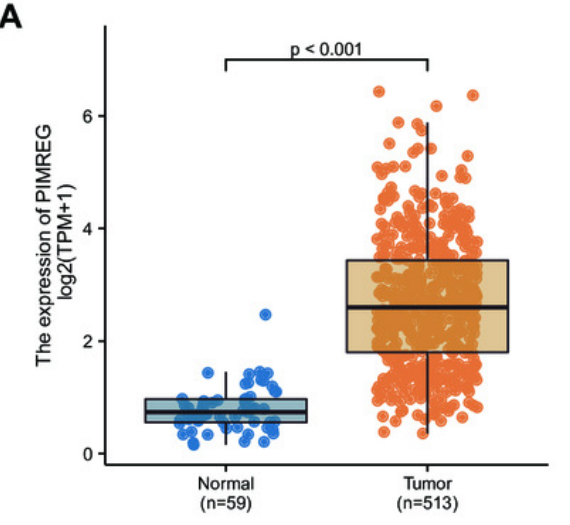

B

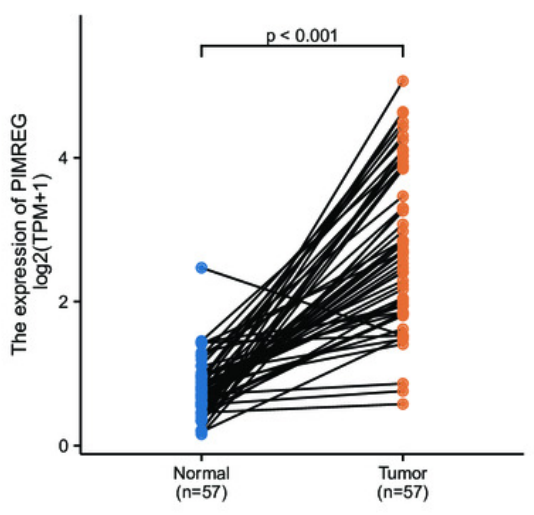

C

追 TCGA_Normal 追 TCGA_Tumor

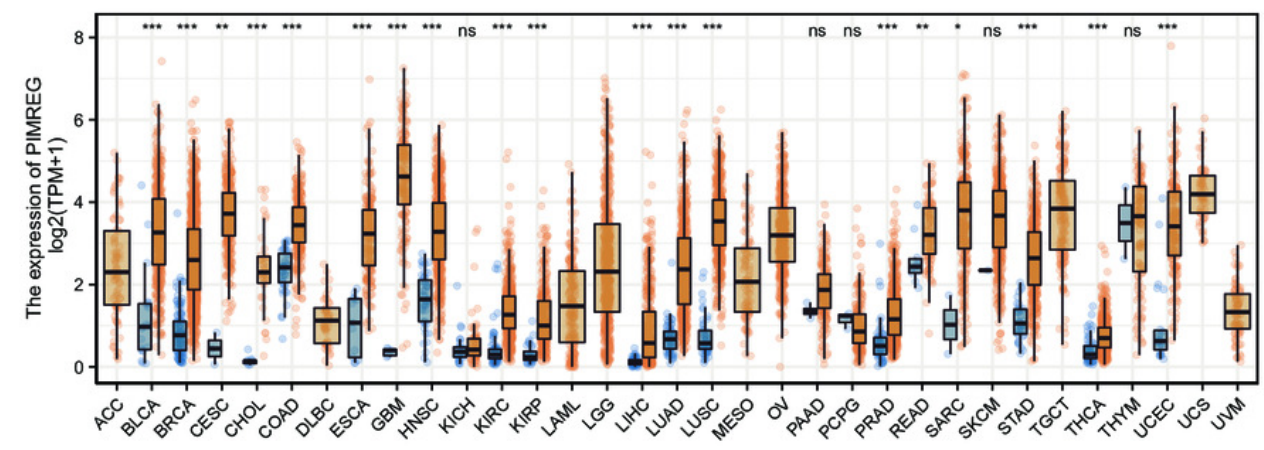


Figure 2

Correlation between PIMREG expression and clinicopathological features.

(A) Clinical stage. (B) Tclassification. (C) N classification. (D) M classification. (E) TP53 status.

(F) smoke. 

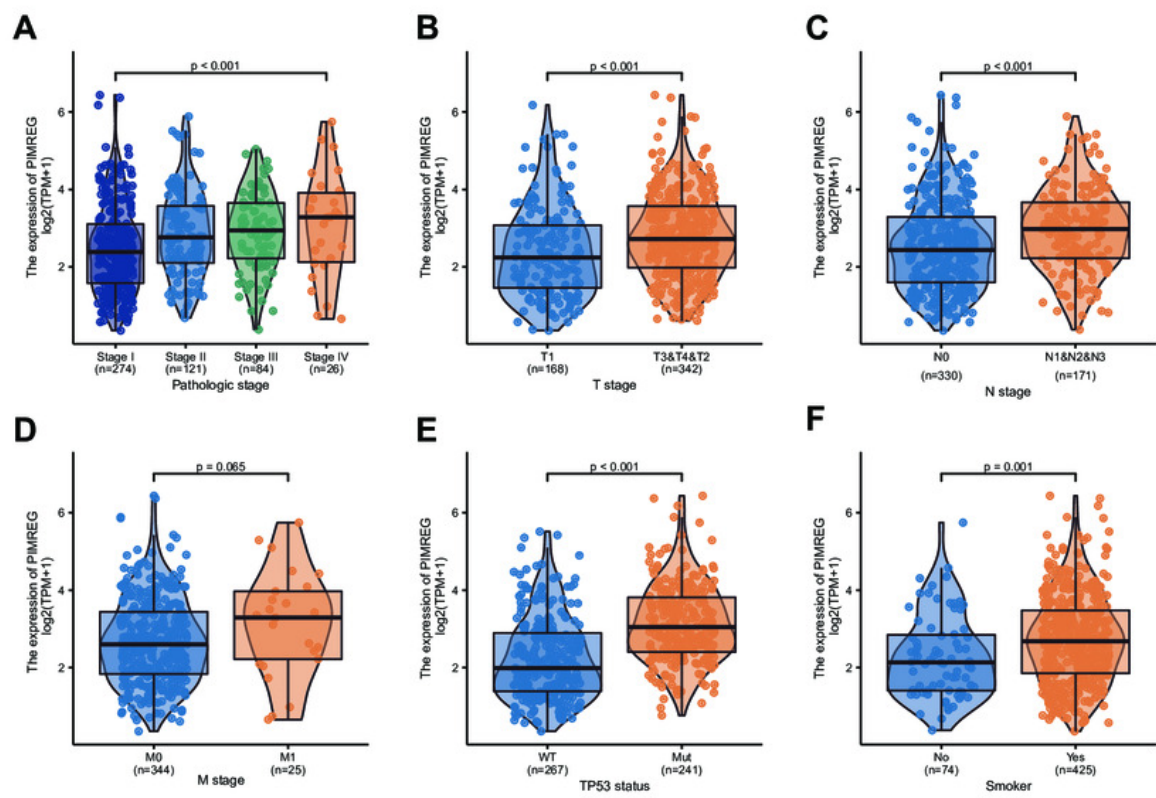

F

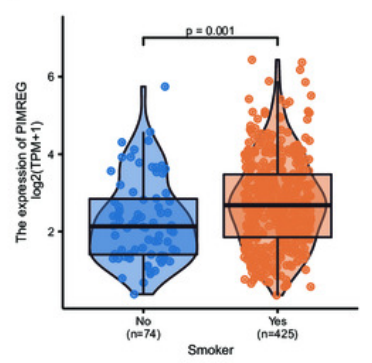


Figure 3

The overall survival, progression-free survival and relapse-free survival rates in PIMREG high and low patient groups.
(A) Overall survival. (B) Disease-Specific Survival (DSS). (C) Progression-Free Interval (PFI).
(D) ROC. 

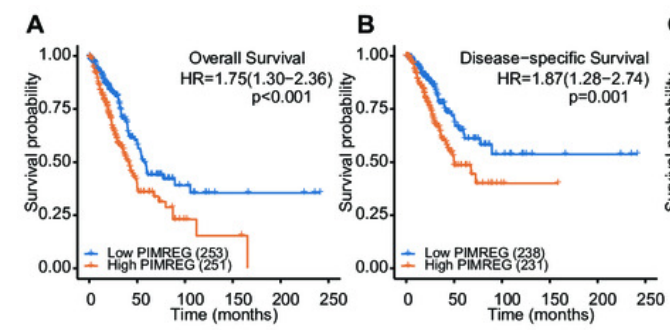

C

D

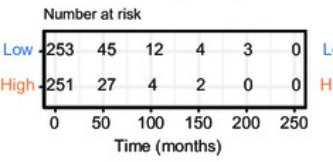

Number at risk
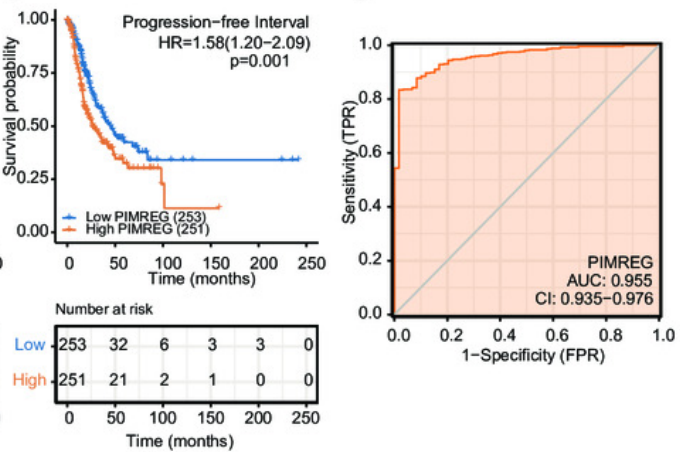


\section{Figure 4}

Subgroup analysis.

(A) The Kaplan-Meier curves for age < 65 years subgroup. (B) The Kaplan-Meier curves for age $\geq 65$ years subgroup. (C) The Kaplan-Meier curves for the male subgroup. (D) The Kaplan-Meier curves for the female subgroup. (E) The Kaplan-Meier curves for the left of anatomic neoplasm subdivision subgroup. F, The Kaplan-Meier curves for the right of anatomic neoplasm subdivision subgroup. 

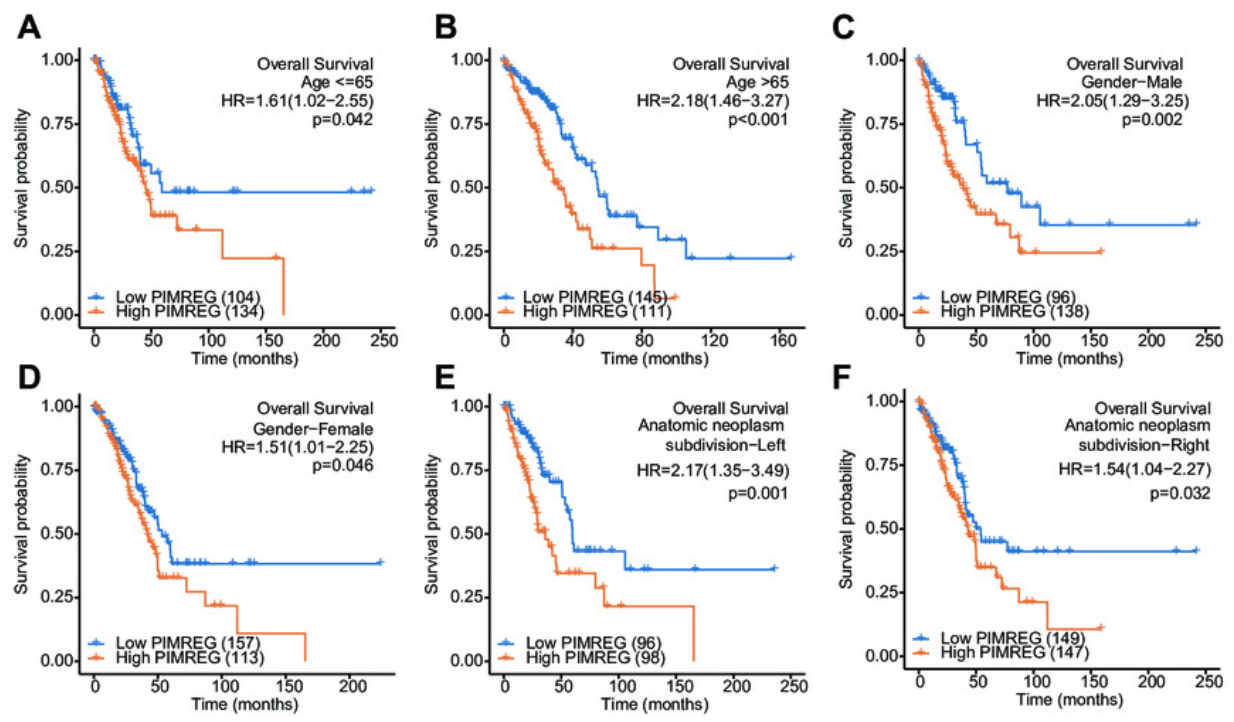
Figure 5

The role of PIMREG in predicting the prognosis of LUAD patients.

(A) Terminology diagram. (B) Calibration curve. 
A

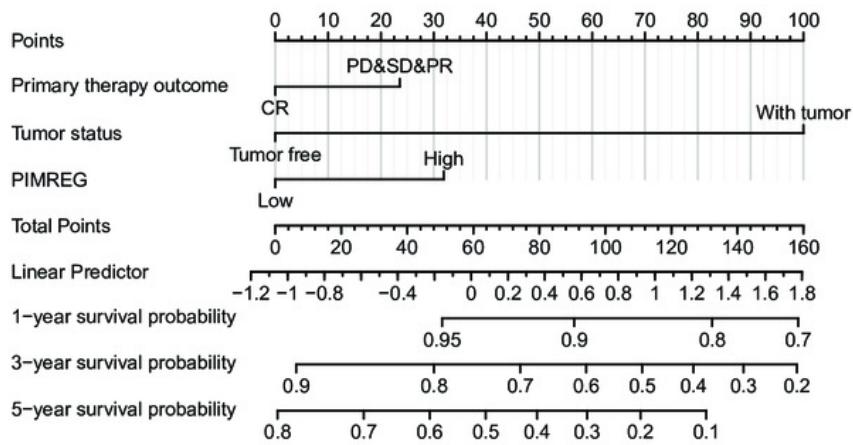

B

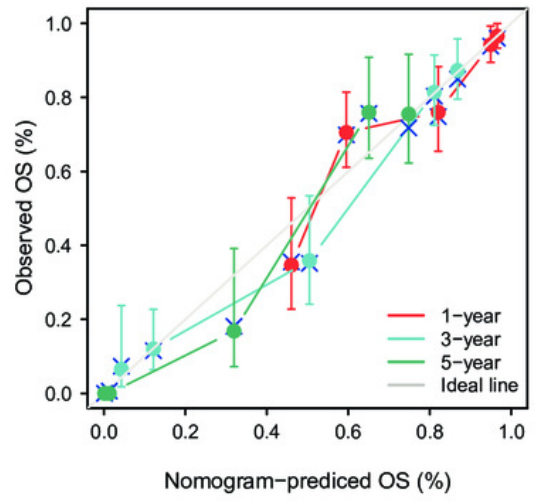


Figure 6

GSEA analyses in LUAD patients with high expression of PIMREG compared with the ones with low expression.

NES: normalized enrichment score; ADJ: adjusted; FDR: false discovery rate. 

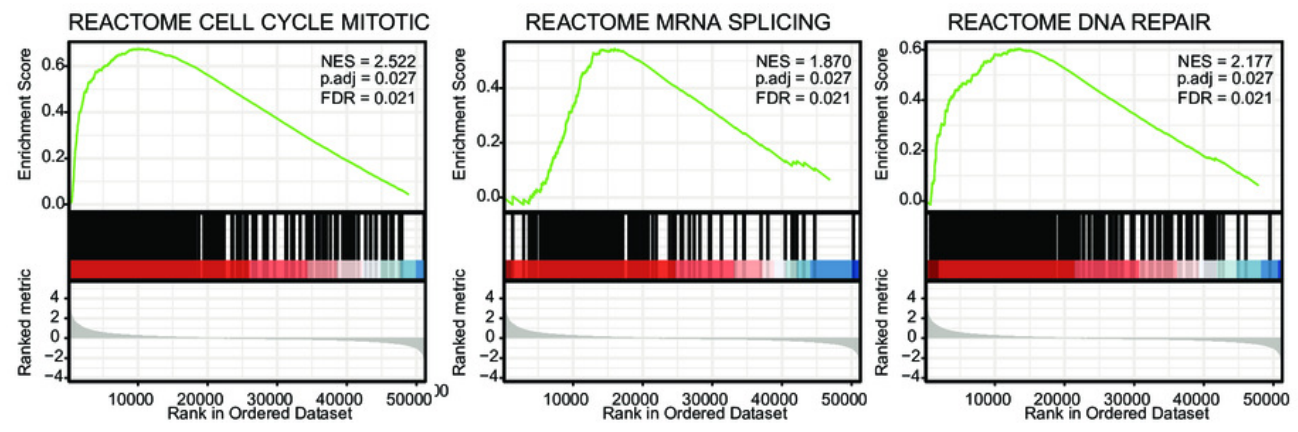

REACTOME SIGNALING BY RHO

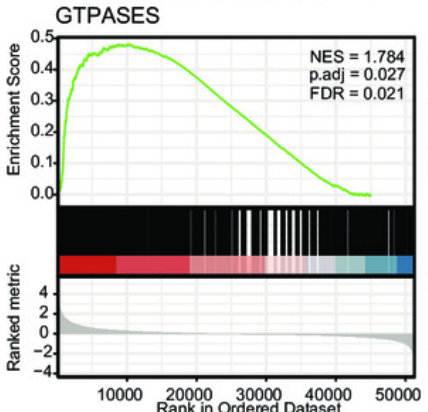

REACTOME TRANSCRIPTIONAL REGULATION BY TP53

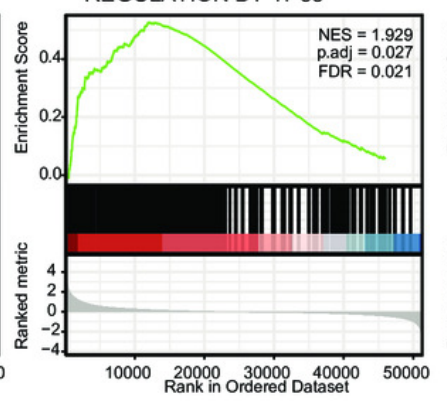

REACTOME TRANSLATION

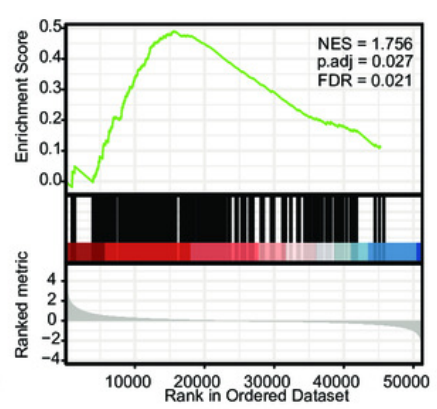


Figure 7

The correlation between immune infiltrating level and PIMREG expression in LUAD. 

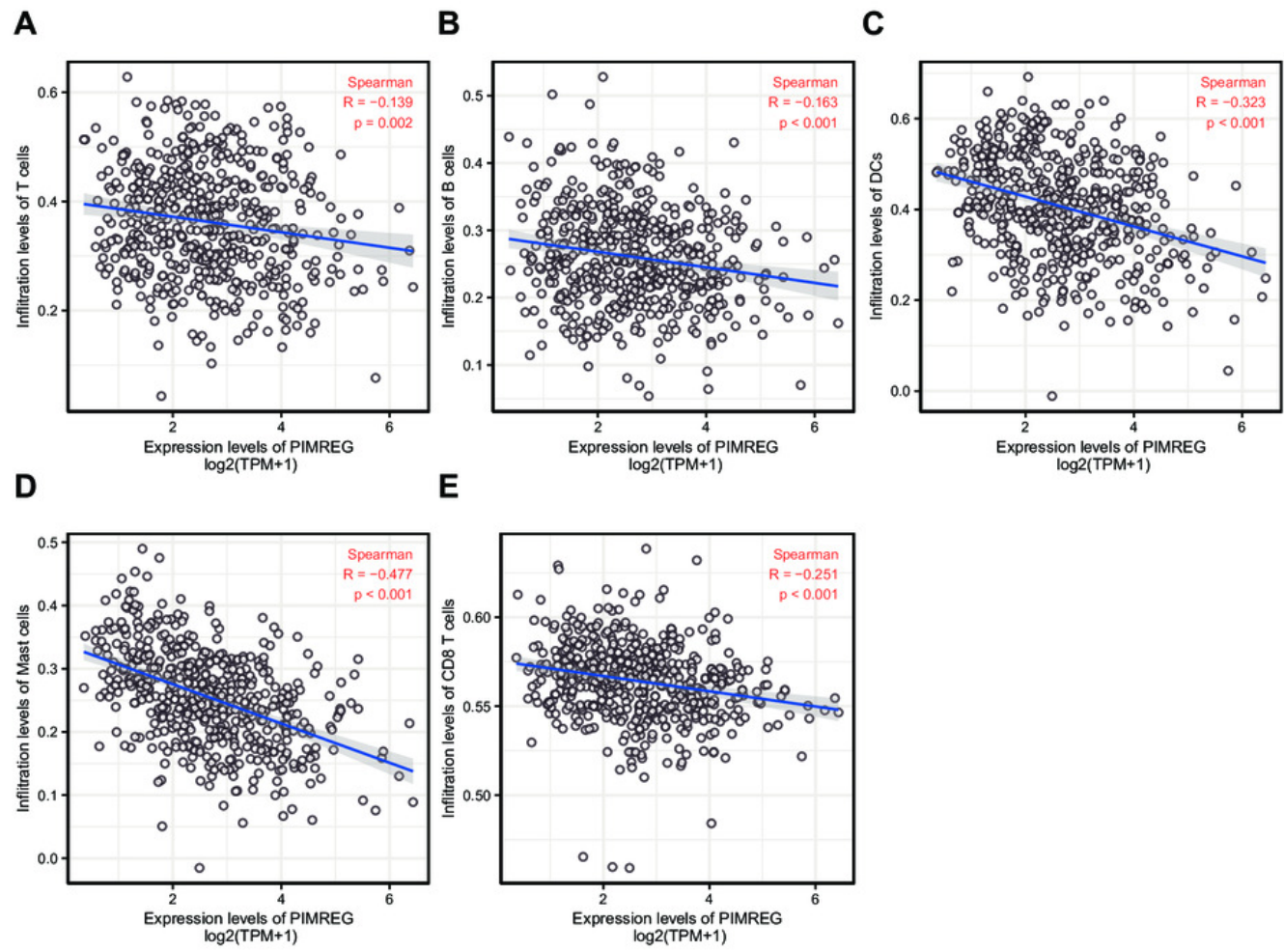
Figure 8

The expression level of PIMREG in LUAD cell lines and normal lung epithelial cell line 2B. 


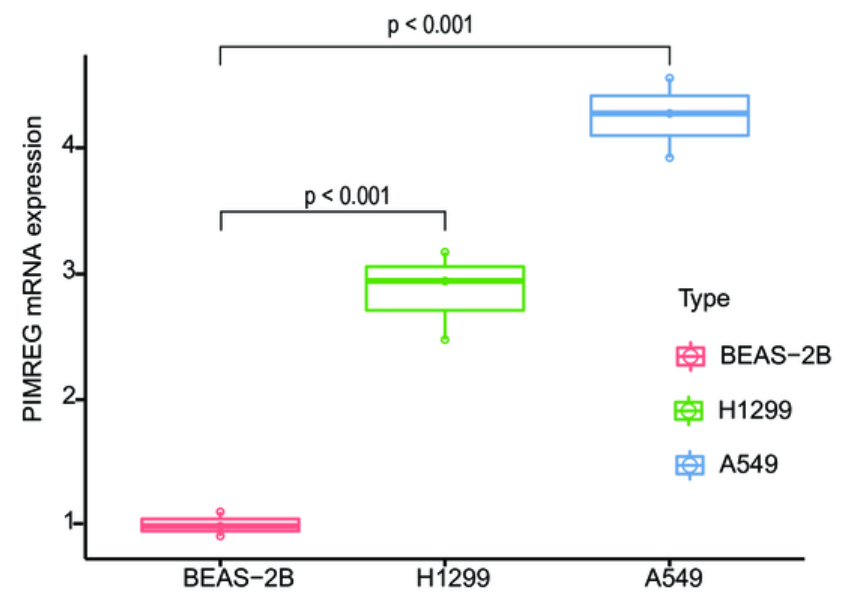

